Article

\title{
The Quantum-Classical Transition as an Information Flow
}

Andres M. Kowalski ${ }^{1,2}$, Maria T. Martin ${ }^{2,3}$, Luciano Zunino ${ }^{3,4,5, \dagger}$, Angelo Plastino ${ }^{2,3, \star}$ and $^{\text {and }}$ Montserrat Casas ${ }^{6}$

${ }^{1}$ Comisión de Investigaciones Científicas (CIC), Argentina

${ }^{2}$ Instituto de Física (IFLP-CCT-Conicet), Fac. de Ciencias Exactas, Universidad Nacional de La Plata, C.C. 727,1900 La Plata, Argentina

${ }^{3}$ Argentina's National Research Council (CONICET), Argentina

${ }^{4}$ Centro de Investigaciones Ópticas (CONICET La Plata - CIC), C.C. 3, 1897 Gonnet, Argentina

${ }^{5}$ Departamento de Ciencias Básicas, Facultad de Ingeniería, Universidad Nacional de La Plata (UNLP), 1900 La Plata, Argentina

${ }^{6}$ Departament de Física and IFISC, Universitat de les Illes Balears, 07122 Palma de Mallorca, Spain

† Instituto de Física Interdisciplinar y Sistemas Complejos (IFISC) CSIC-UIB, Campus Universitat de les Illes Balears, E-07122 Palma de Mallorca, Spain.

^ Author to whom correspondence should be addressed; E-Mail: plastino@ fisica.unlp.edu.ar.

Received: 21 October 2009; in revised form: 9 December 2009/ Accepted: 11 December 2009 / Published: 26 January 2010

\begin{abstract}
We investigate the classical limit of the semiclassical evolution with reference to a well-known model that represents the interaction between matter and a given field. This is done by recourse to a special statistical quantifier called the "symbolic transfer entropy". We encounter that the quantum-classical transition gets thereby described as the sign-reversal of the dominating direction of the information flow between classical and quantal variables.
\end{abstract}

Keywords: transfer entropy; Bandt and Pompe method; semiclassical theories; quantum chaos

Classification: PACS 89.70.Cf (Entropy and other measures of information), 05.45.Tp (Time series analysis), 03.65.Sq (Semiclassical theories and applications), 05.45.Mt (Quantum chaos; semiclassical methods) 


\section{Introduction}

The synchronization phenomenon is the subject of much work nowadays and attracts attention from various field of science, engineering, and social behavior [1]. In particular, the coupling between dynamical systems is of particular interest as it can be encountered in a number of manners. To investigate the associated interactions involves the detection and quantification of the strength and direction (or asymmetry) of the pertinent couplings and major effort has revolved around the task of ascertaining directional couplings. Information-theoretic approaches become of importance in such respect. One of such treatments is of paramount interest for us here, related to the quantity called "transfer entropy" (TE) [2], which can quantify statistical coherence among systems evolving in time. The corresponding approach was designed so as to overcome difficulties encountered using the standard time delayed mutual information, which exhibits problems in distinguishing between information that is actually exchanged from shared information due to common history and input signals [3]. The TE ignores these influences by appropriately conditioning associated transition probabilities [2]. One is thus able to distinguish effectively driving from responding elements and to detect asymmetry in the interaction of subsystems. Interesting progress is advanced in [3] by estimating the transfer entropy by recourse to a symbolization-technique, tantamount to a sort of coarse graining that facilitates accessible manipulation. One then speaks of symbolic TE (STE) and finds that it is a robust and computationally fast method to quantify the dominating direction of information flow between time series from coupled systems.

In this work we apply the STE to the classical limit of quantum mechanics (CLQM) that, contrary to the widespread belief, remains an open problem since the problem of the emergence of classical mechanics from quantum mechanics is by no means solved. In spite of many results on the $\hbar \rightarrow 0$ asymptotics, it is not yet clear how to explain the classical motion of macroscopic bodies within the standard quantum mechanics. In this paper we shall analyze, via the STE, a special case of evolution from quantum to classical behavior [4] in the framework of a well-known semi-classical model that represents the interaction of matter with a given field $[5,6]$.

\section{The CLQM for a Special Semi-Classical Model}

Since the introduction of the decoherence concept in the early 1980s by Zeh, Zurek, and other authors like Habib [7-11], the emergence of the classical world from Quantum Mechanics has been a subject of much interest. Among the associated issues one can mention the emergence of classical dynamics, specially classical chaos, in quantum systems through continuous measurement by Habib, Bhattacharya, Ghose, and Jacobs, among others [12,13] and the "decoherent histories approach" by Gisin, Brun, Halliwell, and Percival [14-17]. Additionally, authors like Everitt, who explore the quantum-classical crossover in the behavior of a quantum field mode [18] and the chaotic-like and non-chaotic-like behavior in nonlinear quantum systems [19] are of certain interest. Also deserve mention Ralph [20], Greenbaum [21] and Lifshitz [22].

Quite a bit of quantum insight is to be gained from semiclassical perspectives. Several methodologies are available (WKB, Born-Oppenheimer approach, etc.). The model of References [5, 6, 23] considers two interacting systems: one classical, the other quantal. This makes sense whenever the quantum effects 
of one of the two systems are negligible in comparison to those of the other one. Examples can be readily found. We can just mention Bloch equations [24], two-level systems interacting with an electromagnetic field within a cavity [25-27], collective nuclear motion [28], etc.

We have investigated the classical limit of a semiclassical model containing both classical and quantum degrees of freedom in Reference [23]. In contrast to what was done in the above mentioned papers via a master equation for the density operator $[10,11,16]$, or by recourse to equivalent stochastic equations for pertinent expectation values $[12,13]$, we consider a simplified scheme in which the interaction with the environment is simulated by classical variables. Here the classical limit is obtained whenever one satisfies the relation given by Equation (4) for the total energy and the invariant $I$ (see Equation (5)), related to the uncertainty principle.

Thus, we deal with a special bipartite system that represents the zero-th mode contribution of a strong external field to the production of charged meson pairs [6, 23], whose Hamiltonian reads

$$
\hat{H}=\frac{1}{2}\left(\frac{\hat{p}^{2}}{m_{q}}+\frac{P_{A}^{2}}{m_{c l}}+m_{q} \omega^{2} \hat{x}^{2}\right)
$$

where $i) \hat{x}$ and $\hat{p}$ are quantum operators, ii) $A$ and $P_{A}$ classical canonical conjugate variables and iii) $\omega^{2}=\omega_{q}{ }^{2}+e^{2} A^{2}$ is an interaction term that introduces nonlinearity, $\omega_{q}$ being a frequency. The quantities $m_{q}$ and $m_{c l}$ are masses, corresponding to the quantum and classical systems, respectively. As shown in Reference [4], in dealing with Equation (1) one faces an autonomous system of nonlinear coupled equations

$$
\begin{array}{lll}
\frac{d\left\langle\hat{x}^{2}\right\rangle}{d t}=\frac{\langle\hat{L}\rangle}{m_{q}}, & \frac{d\left\langle\hat{p}^{2}\right\rangle}{d t}=-m_{q} \omega^{2}\langle\hat{L}\rangle, & \frac{d\langle\hat{L}\rangle}{d t}=2\left(\frac{\left\langle\hat{p}^{2}\right\rangle}{m_{q}}-m_{q} \omega^{2}\left\langle\hat{x}^{2}\right\rangle\right), \\
\frac{d A}{d t}=\frac{P_{A}}{m_{c l}}, & \frac{d P_{A}}{d t}=-e^{2} m_{q} A\left\langle\hat{x}^{2}\right\rangle, &
\end{array}
$$

where $\hat{L}=\hat{x} \hat{p}+\hat{p} \hat{x}$. The system of Equation (2) follows immediately from Ehrenfest's relations [4]. To study the classical limit we also need to consider the classical counterpart of the Hamiltonian given by Equation (1)

$$
H=\frac{1}{2}\left(\frac{p^{2}}{m_{q}}+\frac{P_{A}{ }^{2}}{m_{c l}}+m_{q} \omega^{2} x^{2}\right)
$$

where all the variables are classical. Recourse to Hamilton's equations allows one to find the classical version of Equation (2) (see Reference [4] for further details). These equations are identical in form to Equation (2) after suitable replacement of quantum mean values by classical variables, i.e., $\left\langle\hat{x}^{2}\right\rangle \Rightarrow x^{2}$, $\left\langle\hat{p}^{2}\right\rangle \Rightarrow p^{2}$ and $\langle\hat{L}\rangle \Rightarrow L=2 x p$. The classical limit is obtained by letting the "relative energy"

$$
E_{r}=\frac{|E|}{I^{1 / 2} \omega_{q}} \rightarrow \infty
$$

where $E$ is the total energy of the system and $I$ is an invariant of the motion described by the system of equations previously introduced (Equation (2)), related to the Uncertainty Principle

$$
I=\left\langle\hat{x}^{2}\right\rangle\left\langle\hat{p}^{2}\right\rangle-\frac{\langle\hat{L}\rangle^{2}}{4} \geq \frac{\hbar^{2}}{4}
$$

A classical computation of $I$ yields $I=x^{2} p^{2}-L^{2} / 4 \equiv 0$. Thus, $I$ vanishes when it is evaluated using the classical variables $A$ and $P_{A}$, for all $t$, i.e., $I\left(A, P_{A}\right)=0$, a fact that exhibits the self-consistency of 
our methodology. A measure of the degree of convergence between classical and quantum results in the limit of Equation (4) is given by the norm $\mathcal{N}$ of the vector $\Delta u=u-u_{c l}$ [4]

$$
\mathcal{N}_{\Delta u}=\left|u-u_{c l}\right|
$$

where the three components vector $u=\left(\left\langle\hat{x}^{2}\right\rangle,\left\langle\hat{p}^{2}\right\rangle,\langle\hat{L}\rangle\right)$ is the "quantum" part of the solution of the system defined by Equation (2) and $u_{c l}=\left(x^{2}, p^{2}, L\right)$ its classical counterpart.

A detailed study of this model, was performed in References [4, 23]. The main results of these references, pertinent for our discussion, can be succinctly detailed as follows: in plotting diverse dynamical quantities as a function of $E_{r}$ (as it grows from unity to $\infty$ ), one finds an abrupt change in the system's dynamics for a special value of $E_{r}$, to be denoted by $E_{r}{ }^{c l}$. From this value onwards, the pertinent dynamics starts converging to the classical one. It is thus possible to assert that $E_{r}{ }^{c l}$ provides us with an indicator of the presence of a quantum-classical "border". The zone

$$
E_{r}<E_{r}^{c l}
$$

corresponds to the semi-quantal regime investigated in Reference [23]. This regime, in turn, is characterized by two different sub-zones [23]. One of them is an almost purely quantal one, in which the microscopic quantal oscillator is just slightly perturbed by the classical one, and the other section exhibits a transitional nature (semi-quantal). The border between these two sub-zones can be well characterized by a "signal" value $E_{r}{ }^{\mathcal{P}}$. A significant feature of this point resides in the fact that, for $E_{r} \geq E_{r}{ }^{\mathcal{P}}$, chaos is always found. The relative number of chaotic orbits (with respect to the total number of orbits) grows with $E_{r}$ and tends to unity for $E_{r} \rightarrow \infty$ [23].

Thus, as $E_{r}$ grows from $E_{r}=1$ (the "pure quantum instance") to $E_{r} \rightarrow \infty$ (the classical situation), a significant series of morphology changes is detected, specially in the transition-zone $\left(E_{r}{ }^{\mathcal{P}} \leq E_{r} \leq E_{r}{ }^{c l}\right)$. The concomitant orbits exhibit features that are not easily describable in terms of Equation (6), which is a global measure of the degree of convergence in amplitude (of the signal). What one needs instead is a statistical type of characterization, as that described in References [29-31], involving the notions of entropy and statistical complexity. Both quantifiers can be evaluated in various ways as, for instance, by employing the wavelet approach (see References [29, 30] and references therein) or the Bandt and Pompe method (see Reference [31] and references therein). These two statistical quantifiers are able to adequately identify the properties of the three zones that enter the quantum-classical evolution (as $E_{r}$ varies). However, the intermediate-transition zone needs still more detailed analysis that will be shown below to be provided by recourse to the symbolic transfer entropy.

\section{Symbolic Transfer Entropy}

In order to understand the flow of information between two different time series Schreiber [2] introduced the transfer entropy concept. This quantifier allows to determine driving and responding elements and to detect asymmetry in the interaction between two systems. Let us consider $x_{i}$ and $y_{i}, i=1, \ldots, N$, two realizations of the systems $X$ and $Y$. The transfer entropy, defined as the Kullback-Leibler entropy between the conditional probabilities $p\left(x_{i+1} \mid x_{i}, y_{i}\right)$ and $p\left(x_{i+1} \mid x_{i}\right)$, measures the deviation from the generalized Markov property

$$
p\left(x_{i+1} \mid x_{i}, y_{i}\right)=p\left(x_{i+1} \mid x_{i}\right)
$$


This deviation quantifies the influence of the system $Y$ on $X$. Observe that the transfer entropy is explicitly nonsymmetric under the changes of $x_{i}$ and $y_{i}$. This tool has been applied for assessing causality relations in different fields like econophysics [32-34] and chaotic communications [35]. It was also shown that the Schreiber's definition of TE coincides with the standard definition of the conditional mutual information [36, 37].

Unfortunately, due to several drawbacks such as the high amount of data required and its sensitivity to noise contributions, this information theoretic measure is very hard to estimate in practical analyses. More recently, Staniek and Lehnertz [3] proposed a modified version of the transfer entropy, the symbolic transfer entropy, based on the Bandt and Pompe symbolization technique [38]. The new symbolic series are obtained by reordering the amplitude values of the original time series $x_{i}$ and $y_{i}$. Consider the sequence of amplitude values associated to the time series $x_{i}$ with embedding dimension $D>1$ and time delay $\tau$ given by

$$
x_{i} \mapsto\left(x_{i-(D-1) \tau}, x_{i-(D-2) \tau}, \ldots, x_{i-\tau}, x_{i}\right)
$$

To each time $i$ we are assigning a $D$-dimensional vector that results from the evaluation of the time series at times $i, i-\tau, \ldots, i-(D-1) \tau$. Clearly, the greater the $D$ value, the more information about the past is incorporated into the ensuing vectors. By the ordinal pattern of order $D$ related to the time $i$ we mean the permutation $\hat{x}_{i}=\left(r_{0}, r_{1}, \cdots, r_{D-1}\right)$ of $(0,1, \cdots, D-1)$ defined by

$$
x_{i-r_{D-1} \tau} \leq x_{i-r_{D-2} \tau} \leq \cdots \leq x_{i-r_{1} \tau} \leq x_{i-r_{0} \tau}
$$

In this way the vector defined by Equation (9) is converted into a unique symbol $\hat{x}_{i}$. Further details about the Bandt and Pompe method can be found in References $[39,40]$. For all the $D$ ! possible permutations $\hat{x}_{i}$ of order $D$, their associated relative frequencies can be naturally computed by

$$
p\left(\hat{x}_{i}\right)=\frac{\sharp\left\{i \mid 1+(D-1) \tau \leq i \leq N \text { and } i \text { has ordinal pattern } \hat{x}_{i}\right\}}{N-(D-1) \tau}
$$

where $\sharp$ is the cardinality of the set—roughly speaking, the number of elements in it. Thus, a permutation probability distribution $P_{x}=\left\{p\left(\hat{x}_{i}\right), i=1, \ldots, D\right.$ ! $\}$ is obtained from the time series $x_{i}$ [42]. Obviously the same can be done for $y_{i}$. Given these permutation probability distributions the symbolic transfer entropy (STE) is defined as

$$
T_{Y, X}^{S}=\sum p\left(\hat{x}_{i+\delta}, \hat{x}_{i}, \hat{y}_{i}\right) \log \frac{p\left(\hat{x}_{i+\delta} \mid \hat{x}_{i}, \hat{y}_{i}\right)}{p\left(\hat{x}_{i+\delta} \mid \hat{x}_{i}\right)}
$$

where the sum runs over all possible permutations and $\delta$ denotes a time step. The joint probability $p\left(\hat{x}_{i+\delta}, \hat{x}_{i}, \hat{y}_{i}\right)$ is the joint relative frequency of the three events $\hat{x}_{i+\delta}, \hat{x}_{i}$ and $\hat{y}_{i}$. The conditional probabilities $p\left(\hat{x}_{i+\delta} \mid \hat{x}_{i}, \hat{y}_{i}\right)$ and $p\left(\hat{x}_{i+\delta} \mid \hat{x}_{i}\right)$ are given by

$$
p\left(\hat{x}_{i+\delta} \mid \hat{x}_{i}, \hat{y}_{i}\right)=p\left(\hat{x}_{i}, \hat{y}_{i}, \hat{x}_{i+\delta}\right) / p\left(\hat{x}_{i}, \hat{y}_{i}\right)
$$

and

$$
p\left(\hat{x}_{i+\delta} \mid \hat{x}_{i}\right)=p\left(\hat{x}_{i}, \hat{x}_{i+\delta}\right) / p\left(\hat{x}_{i}\right)
$$

respectively. The symbolic transfer entropy $T_{Y, X}^{S}$ quantifies the flow of information from system $Y$ to $X$. $T_{X, Y}^{S}$ is defined in the same way. Finally, the directionality index $T^{S}=T_{X, Y}^{S}-T_{Y, X}^{S}$ allows us to determine the dominating direction of information flow between the two systems $X$ and $Y$. If $T^{S}>0$, $X$ will be the driving system and $Y$ the responding one. On the other hand this quantifier will be negative for $Y$ driving $X$. 


\section{Results}

Remember that the CLQM-model we are dealing with here is represented by five non-linear coupled equations (Equation (2)). A correct reconstruction of the attractor associated with the integration of this system is achieved using an embedding dimension $D=5$. We will deal with vectors with components of $N=50,000$ data-points each one, for each orbit. Notice that the condition $N \gg D$ ! is satisfied. We take the time delay $\tau=1$ as it is usually chosen [38]. In obtaining our numerical results we chose $m_{q}=m_{c l}=\omega_{q}=e=1$ for the system's parameters. For the initial conditions needed to tackle our system (Equation (2)) we took $E=0.6$, i.e., we fixed $E$ and then varied $I$ so as to obtain our different $E_{r}$-values. Additionally, we set $\langle L\rangle(0)=L(0)=0, A(0)=0$ (both in the quantum and the classical instances), and $\left\langle x^{2}\right\rangle(0)$ taking values in the interval $x^{2}(0)<\left\langle x^{2}\right\rangle(0) \leq 0.502$, with $x^{2}(0)=0.012$. The data-points used to evaluate the probability distribution $p$ in Equation (12) are given by both, the solutions of Equation (2) and its classical counterpart. Finally, a time step $\delta=1$ was used in our calculations.

We will here choose $X$, in Equation (12), as representing the series of values pertaining to a quantum system's variable, while $Y$ does the same classically. Figures 1 and 2 display the quantities $T^{S}=T_{q, c}^{S}-T_{c, q}^{S}, T_{q, c}^{S}$ and $T_{c, q}^{S}$ vs. $E_{r}$, taking $q \equiv\left\langle x^{2}\right\rangle$ and $c \equiv A$. Instead, in Figure 3 we have $q \equiv\left\langle x^{2}\right\rangle$ and $c \equiv P_{A}$. Similar results ensue if other pairs of quantum-classical variables are selected, that are not shown on space-saving grounds.

We pass now to consider what happens when $E_{r}$ varies moving leftward from $+\infty$. In the fully classical case ( $I=0), A-P_{A}$ "lead" [43] the remaining variables of the system of classical equations $\left(x^{2}, p^{2}, L\right)$. For large $E_{r}$ 's $\left(I \neq 0\right.$ but small) the classical variables $A-P_{A}$ "still dominate" and lead the quantal $\left(\left\langle x^{2}\right\rangle,\left\langle p^{2}\right\rangle,\langle\hat{L}\rangle\right)$-ones. See Figure 1 for the $A-\left\langle x^{2}\right\rangle$-scenario. This plot, and also Figure 2, are representative of our process. As $E_{r}$ diminishes further and reaches the particular value $E_{r}=E_{r}^{M}=$ 6.81 where $T^{S}=0$ (Figure 2), the behavior changes. From then on, the quantum mean value $\left\langle x^{2}\right\rangle$ begins to dominate. As $E_{r}$ decreases (or $I$ grows) the influence of the Uncertainty Principle becomes stronger, thus we conclude that it is the responsible for the change. At $E_{r}=E_{r}^{M}$ the Uncertainty Principle becomes important enough and the quantum variable suddenly begins to drive the process (Figure 2). It dominates until, for $E_{r}=1$, we again get $T^{S}=0$. This last result is to be expected, since for $E_{r}=1$ the quantum system acquires all the energy $E=I^{1 / 2} \omega_{q}$ and the classical one gets located at the fixed point $\left(A=0, P_{A}=0\right)$ [23]. Since $A=0$ the two system become decoupled and no information flows between them. Note in Figure $2 \mathrm{~b}$ ) that $T_{q, c}^{S}$ and $T_{c, q}^{S}$ practically vanish. Small biases are obtained due to finite-sample effects. If we increase the series' size these errors diminish. Anyway, if $E_{r} \simeq 1, T_{q, c}^{S}=T_{c, q}^{S}$ and no error is found in $T^{S}$ [3]. Figure 3 depicts a similar behavior, the variables now being $\left\langle x^{2}\right\rangle$ and $P_{A}$. Any classical-quantal pair of variables will exhibit the same behavior.

As it should be expected, in the quantum zone quantal variables predominate, and vice versa in the classical region. On the other hand, we note that at the symmetric-flow point $E_{r}=E_{r}^{M}$, the Statistical Complexity attains a maximum [29-31]. We remind the reader the so called Statistical Complexity reflects on the systems's architecture, being different from zero only if there exist privileged, or more likely states among the accessible ones. It quantifies not only randomness, but the presence of correlational structures as well. The opposite extremes of perfect order and maximal randomness possess no structure to speak of. In between these two special instances, a wide range of possible 
degrees of physical structure exist, degrees that should be reflected in the features of the underlying probability distribution [41]. In Figure 3, the value $T^{S}=0$ does not obtain exactly at $E_{r}^{M}=6.81$ but very near it, with a relative error $\leq 0.12$. Note that $E_{r}^{M}$ divides into two sections the transitional region. The information flow in the leftward subregion is from the quantal variables to the classical ones. In the rightward subregions the information flow reverses its sign. In the former sub-zone the quantum-classical mixture characterizes a phase-space with more non-chaotic than chaotic curves while in the other one things turn around [23].

Figure 1. a) The directionality index $T^{S}$ vs. $E_{r}$ and b) $T_{q, c}^{S}$ and $T_{c, q}^{S}$ vs. $E_{r}$, for a wide $E_{r}$-range. We took $q \equiv\left\langle x^{2}\right\rangle$ and $c \equiv A$. The classical variable $A$ is dominant across most of the range, except for small $E_{r}$-values, for which Uncertainty Principle becomes important enough that the quantal variable $\left\langle x^{2}\right\rangle$ becomes dominant. Note the absolute minimum of $T^{S}$ at $E_{r}{ }^{c l}=21.55264$, beginning of the transition region.
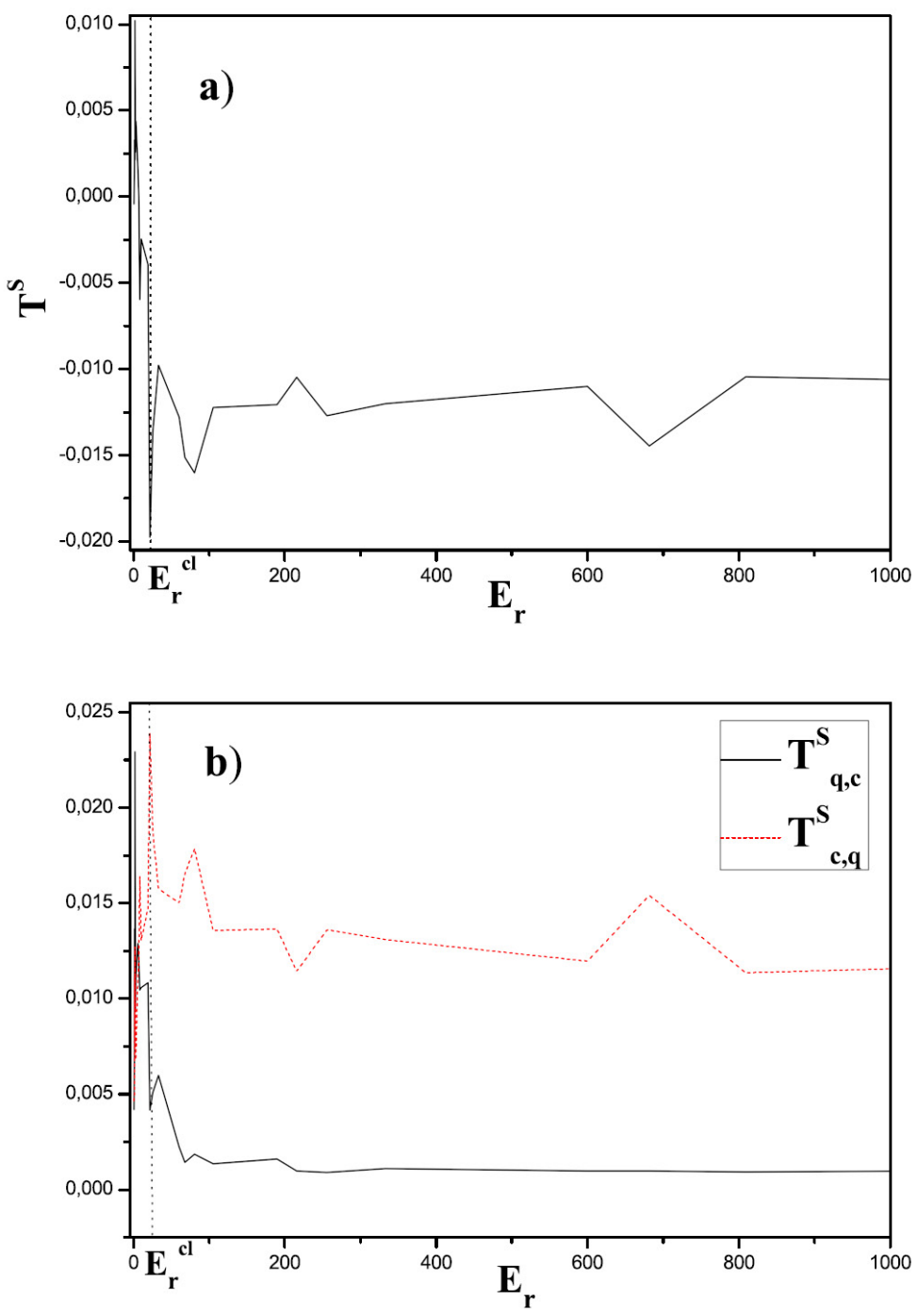
Figure 2. a) The directionality index $T^{S}$ vs. $E_{r}$ and b) $T_{q, c}^{S}$ and $T_{c, q}^{S}$ vs. $E_{r}$, for an $E_{r}$-range that allows to visualize the three zones of the process, i.e., quantal, transitional, and classic, delimited, respectively, by $E_{r}{ }^{\mathcal{P}}=3.3282$, and $E_{r}{ }^{c l}=21.55264$. We took $q \equiv\left\langle x^{2}\right\rangle$ and $c \equiv A$ as in Figure 1. Note the absolute minimum of $T^{S}$ at $E_{r}{ }^{c l}$, the local maximum at $E_{r}{ }^{\mathcal{P}}$, and the absolute maximum close by $\left(E_{r} \simeq 2.2\right)$. Symmetric information flow obtains at $E_{r}^{M}=6.81$ (where the Statistical Complexity attains a maximum), well within the transition region. Classical variable $A$ is the "leading" one from $+\infty$ until this point. For smaller $E_{r}$-values, $\left\langle x^{2}\right\rangle$ becomes dominant.
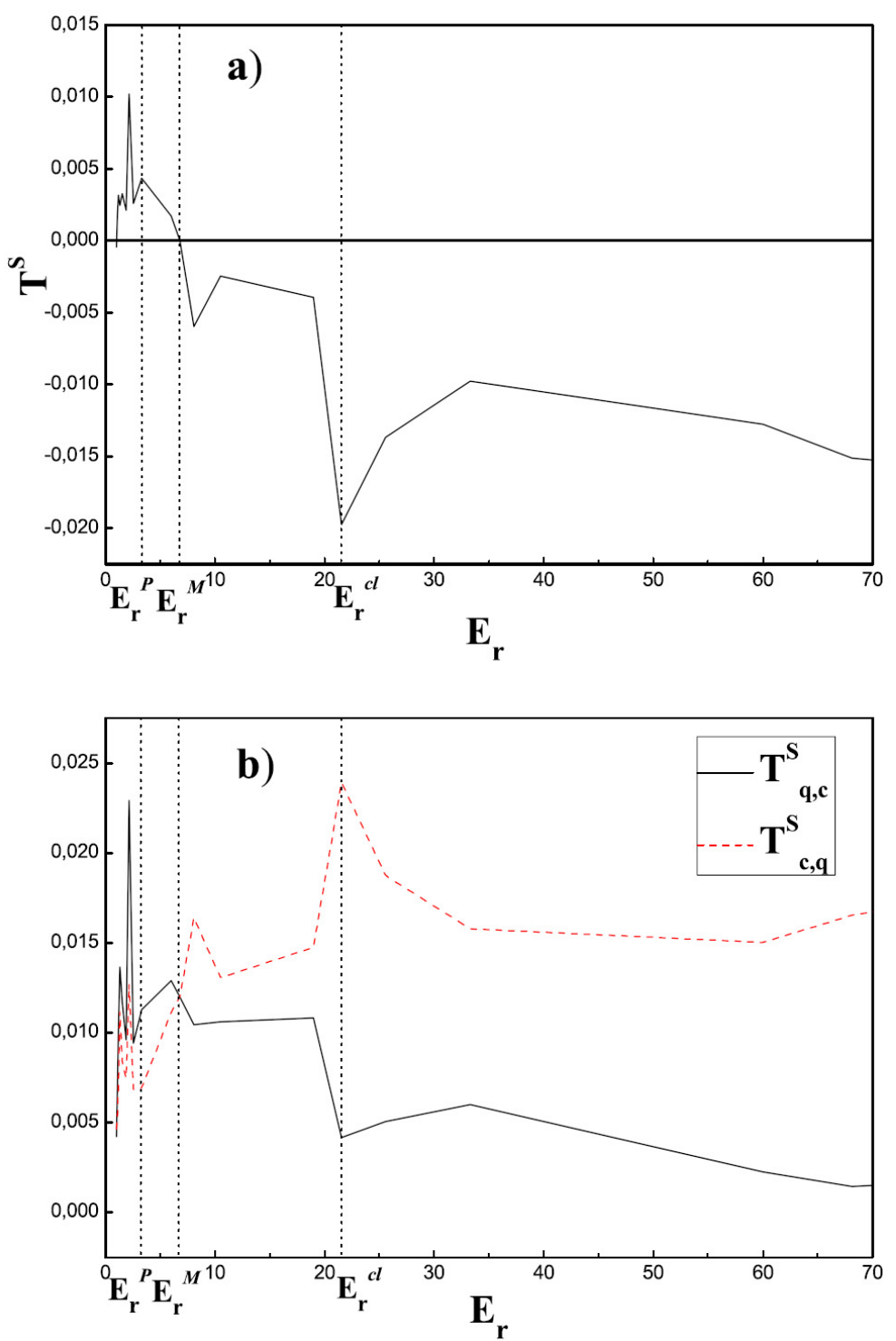

The other interesting points in the $E_{r}$-evolution are $E_{r}{ }^{\mathcal{P}}$ and $E_{r}{ }^{c l}$, at which the transition-zone and the classical one, respectively, begin. At $E_{r}{ }^{c l}=21.55264$ one detects the presence of an absolute minimum of the directional index $T^{S}$ (Figure 1) that measures the quantum-to-classic subsystems flow. This minimum is to be read as representing maximal influence of the classical variables over the quantal ones. In the vicinity of $E_{r}{ }^{\mathcal{P}}=3.3282\left(E_{r} \simeq 2.2\right)$ we encounter an absolute maximum of $T^{S}$ and thus of the quantum-to-classic subsystems flow. There is also a local maximum at $E_{r}{ }^{\mathcal{P}}$ (that becomes 
absolute if $T^{S}$ is evaluated for the pair $\left\langle x^{2}\right\rangle-P_{A}$ (Figure 3)). Thus the transition zone is located within both minimum and maximum values of the classic-to-quantum subsystems flow. The three quantities $T^{S}, T_{q, c}^{S}$, and $T_{c, q}^{S}$ correctly describe the convergence of our quantal results towards the classical ones, as depicted in Figure 1 (fluctuations disappear for large enough values of $E_{r}$ ).

Figure 3. The directionality index $T^{S}$ vs. $E_{r}$ and $T_{q, c}^{S}$ and $T_{c, q}^{S}$ vs. $E_{r}$ (inset). Here we took $q \equiv\left\langle x^{2}\right\rangle$ and $c \equiv P_{A}$. The three stages of the process are visible between $E_{r}{ }^{\mathcal{P}}=3.3282$ and $E_{r}{ }^{c l}=21.55264$. Note the $T^{S}$-absolute maximum at $E_{r}{ }^{\mathcal{P}} . E_{r}^{M}$ is here slightly off the mark (see text).

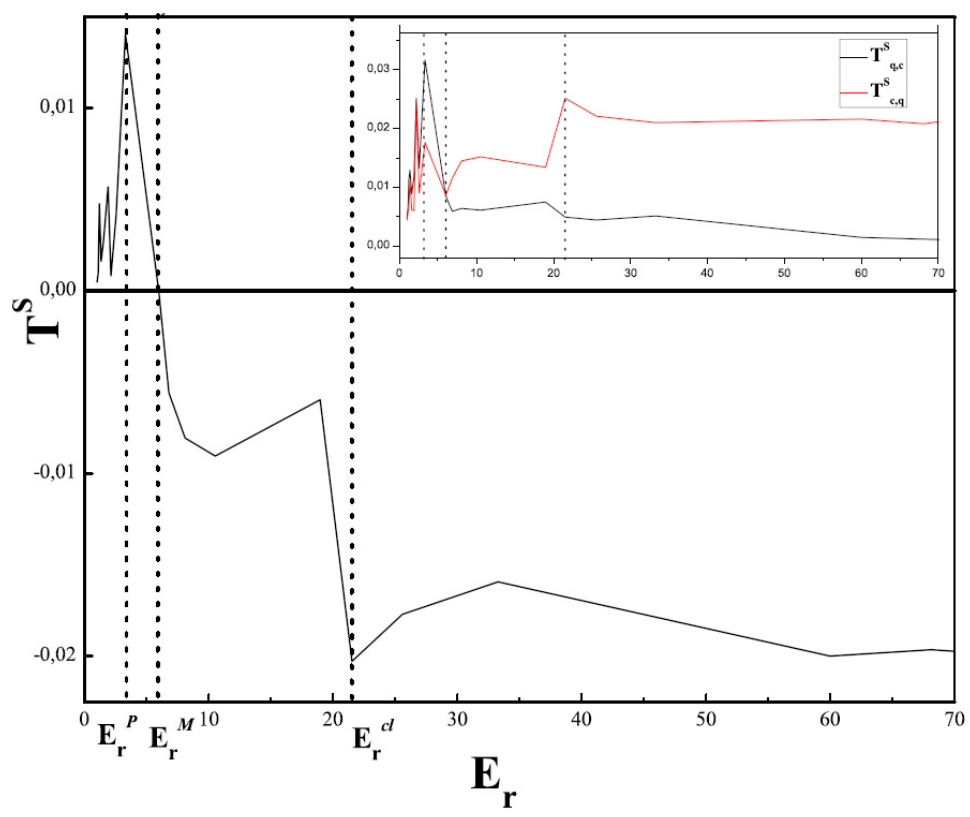

One can also argue that the present results shed new light on what was called the quantal zone in References [29-31]. Strictly speaking, the system of Equation (2) always describes a semiclassical system, for any $E_{r}$. A trivial instance is that of $E_{r}=1$, in which classical-quantal decoupling takes place. In the vicinity of $E_{r}=1$ the system behaves like a slightly perturbed quantum harmonic oscillator. As $E_{r}$ grows, that associated orbits undergo a deformation process, but retain their quasi-periodic nature, as long as $E_{r}$ remains within the quantum zone, however the system of Equation (2) does represent a coupled system, entailing that the evolution of the quantal variables depends upon the classical ones. Thus, it is not a trivial fact that we can speak of a quantum zone. That we properly do is now confirmed in the light of our present results regarding the fact that the quantum variables govern the information flow in this zone.

\section{Conclusions}

In the present communication we have delved into the classical-quantal frontier problem using as a tool the symbolic transfer entropy for investigating the dynamics generated by a semi-classical Hamiltonian that represents the zero-th mode contribution of an strong external field to the production of charged meson pairs [6, 23]. 
The dynamical analysis of the problem performed in Reference [23] specified the highlights of the road towards classicality of the dynamics of the semi-quantum system in question, via a description in terms of the relative energy $E_{r}$ given by Equation (4). As $E_{r}$ grows from $E_{r}=1$ (the "pure quantum instance") to $E_{r} \rightarrow \infty$ ( $I=0$, the classical situation), a significant series of morphology changes are detected in the solutions of the system of nonlinear coupled equations defined by Equation (2). The concomitant process takes place in three stages: quantal, transitional, and classic, delimited, respectively, by special values of $E_{r}$, namely, $E_{r}{ }^{\mathcal{P}}$ and $E_{r}{ }^{c l}$. This dynamical description was also reobtained later via statistical treatments in References [29-31].

Here we have seen that using the notion of symbolic transfer entropy with its directional index $T^{S}$ the problem tackled in these references can be expressed in a different light, namely, as an information flow between classical and quantal variables. We demonstrate that, starting from $E_{r}=\infty$ leftwards, classical variables lead the process until the effects of the Uncertainty Principle become important enough at $E_{r}^{M}$. At this particular value the information flow becomes symmetric and for larger energy values it reverses its sign. We conclude that the Uncertainty Principle is the responsible for this change so that we can associate the flow inversion with the classical-quantal transition. At $E_{r}^{M}$ the statistical complexity becomes maximal [29-31] entailing that symmetry of information flow is tantamount with maximum complexity. We note that ${E_{r}}^{\mathcal{P}}$ and $E_{r}{ }^{c l}$ are, respectively, points of maximal quantal-classical and maximal classical-quantal information-flows. Additionally, the present results shed new light on what was called the quantal zone, as discussed at the end of the preceding section, as therein the quantum variables do govern the information flow.

Finally, we have shown the efficiency of the new information-quantifier called the symbolic transfer entropy and protagonist of the present considerations, validating its physical significance, which should encourage its application to other problems.

\section{Acknowledgements}

The authors wish to thank Matthäus Staniek for help in producing the symbolic transfer entropy algorithm. A. M. Kowalski is supported by CIC of Argentina and L. Zunino by Consejo Nacional de Investigaciones Científicas y Técnicas (CONICET), Argentina. MC was partially supported by the MEC grant No. FIS2008-0781 (Spain).

\section{References and Notes}

1. Pikovsky, A.; Rosenblum, M.; Kurths, J. Synchronization: A Universal Concept in Nonlinear Science; Cambridge University Press: Cambridge, MA, USA, 2001.

2. Schreiber, T. Measuring information transfer. Phys. Rev. Lett. 2000, 85, 461-464.

3. Staniek, M.; Lehnertz, K. Symbolic tranfer entropy. Phys. Rev. Lett. 2008, 100, 15810:1-15810:4.

4. Kowalski, A.M.; Martin, M.T.; Nuñez, J.; Plastino, A.; Proto, A.N. Quantitative indicator for semiquantum chaos. Phys. Rev. A 1998, 58, 2596-2599. 
5. Bonilla, L.L.; Guinea, F. Collapse of the wave packet and chaos in a model with classical and quantum degrees of freedom. Phys. Rev. A 1992, 45, 7718-7728.

6. Cooper, F.; Dawson, J.; Habib, S.; Ryne, R.D. Chaos in time-dependent variational approximations to quantum dynamics. Phys. Rev. E 1998, 57, 1489-1498.

7. Zeh, H.D. Why Bohm's quantum theory? Found. Phys. Lett. 1999, 12, 197-200.

8. Zurek, W.H. Pointer basis of quantum apparatus: Into what mixture does the wave packet collapse? Phys. Rev. D 1981, 24, 1516-1525.

9. Zurek, W.H. Decoherence, einselection, and the quantum origins of the classical. Rev. Mod. Phys. 2003, 75, 715-775.

10. Zurek, W.H.; Habib, S.; Paz, J.P. Coherent states via decoherence. Phys. Rev. Lett. 1993, 70, 1187-1190.

11. Habib, S.; Shizume, K.; Zurek, W.H. Decoherence, chaos, and the correspondence principle. Phys. Rev. Lett. 1998, 80, 4361-4365.

12. Ghose, S.; Alsing, P.; Deutsch, I.; Bhattacharya, T.; Habib, S.; Jacobs, K. Recovering classical dynamics from coupled quantum systems through continuous measurement. Phys. Rev. A 2003, 67, 052102:1-052102:4.

13. Ghose, S.; Alsing, P.; Deutsch, I.; Bhattacharya, T.; Habib, S. Transition to classical chaos in a coupled quantum system through continuous measurement. Phys. Rev. A 2004, 69, 052116:1-052116:8.

14. Diósi, L.; Gisin, N.; Halliwell, J.; Percival, I.C. Decoherent histories and quantum state diffusion. Phys. Rev. Lett. 1995, 74, 203-207.

15. Brun, T.A.; Halliwell, J.J. Decoherence of hydrodynamic histories: A simple spin model. Phys. Rev. D 1996, 54, 2899-2912.

16. Brun, T.A. Continuous measurements, quantum trajectories, and decoherent histories. Phys. Rev. A 2000, 61, 042107:1-042107:17.

17. Halliwell, J.J.; Yearsley, J.M. Arrival times, complex potentials, and decoherent histories. Phys. Rev. A 2009, 79, 062101:1-062101:17.

18. Everitt, M.J.; Munro, W.J.; Spiller, T.P. Quantum-classical crossover of a field mode. Phys. Rev. A 2009, 79, 032328:1-032328:6.

19. Everitt, M.J. Recovery of classical chaotic-like behavior in a conservative quantum three-body problem. Phys. Rev. E 2007, 75, 036217:1-036217:6.

20. Clark, T.D.; Diggins, J.; Ralph, J.F.; Everitt, M.; Prance, R.J.; Prance, H.; Whiteman, R.; Widom, A.; Srivastava, Y.N. Coherent evolution and quantum transitions in a two level model of a SQUID ring. Ann. Phys. N. Y. 1998, 268, 1-30.

21. Greenbaum, B.D.; Habib, S.; Shizume, K.; Sundaram, B. The semiclassical regime of the chaotic quantum-classical transition. Chaos 2005, 15, 033302.

22. Katz, I.; Retzker, A.; Straub, R.; Lifshitz, R. Signatures for a classical to quantum transition of a driven nonlinear nanomechanical resonator. Phys. Rev. Lett. 2007, 99, 040404:1-040404:4.

23. Kowalski, A.M.; Plastino, A.; Proto, A.N. Classical limits. Phys. Lett. A 2002, 297, 162-172.

24. Bloch, F. Nuclear induction. Phys. Rev. 1946, 70, 460-474. 
25. Milonni, P.; Shih, M.; Ackerhalt, J.R. Chaos in Laser-Matter Interactions; World Scientific Publishing Co.: Singapore, 1987.

26. Meystre, P.; Sargent III, M. Elements of Quantum Optics; Springer-Verlag: Berlin, Germany, 1991.

27. Kociuba, G.; Heckenberg, N.R. Controlling the complex Lorenz equations by modulation. Phys. Rev. E 2002, 66, 026205:1-026205:5.

28. Ring, P.; Schuck, P. The Nuclear Many-Body Problem; Springer-Verlag: Berlin, Germany, 1980.

29. Kowalski, A.M.; Martin, M.T.; Plastino, A.; Proto, A.N.; Rosso, O.A. Wavelet statistical complexity analysis of the classical limit. Phys. Lett. A 2003, 311, 180-191.

30. Kowalski, A.M.; Martin, M.T.; Plastino, A.; Rosso, O.A. Entropic non-triviality, the classical limit and geometric-dynamics correlations. Int. J. Mod. Phys. B 2005, 19, 2273-2285.

31. Kowalski, A.M.; Martin, M.T.; Plastino, A.; Rosso, O.A. Bandt-Pompe approach to the classical-quantum transition. Physica D 2007, 233, 21-31.

32. Marschinski, R.; Kantz, H. Analysing the information flow between financial time series. Eur. Phys. J. B 2002, 30, 275-281.

33. Kwon, O.; Yang, J.-S. Information flow between composite stock index and individual stocks. Physica A 2008, 387, 2851-2856.

34. Kwon, O.; Yang, J.-S. Information flow between stock indices. Europhys. Lett. 2008, 82, 68003.

35. Hung, Y.-C.; Hu, C.-K. Chaotic communication via temporal transfer entropy. Phys. Rev. Lett. 2008, 101, 244102:1-244102:4.

36. Hlaváčková-Schindler, K.; Paluš, M.; Vejmelka, M.; Bhattacharya, J. Causality detection based on information-theoretic approaches in time series analysis. Phys. Rep. 2007, 441, 1-46.

37. Paluš, M.; Vejmelka, M. Directionality of coupling from bivariate time series: How to avoid false causalities and missed connections. Phys. Rev. E 2007, 75, 056211:1-056211:14.

38. Bandt, C.; Pompe, B. Permutation entropy: A natural complexity measure for time series. Phys. Rev. Lett. 2002, 88, 174102:1-174102:4.

39. Bandt, C.; Shiha, F. Order patterns in time series. J. Time Ser. Anal. 2007, 28, 646-665.

40. Staniek, M.; Lehnertz, K. Parameter selection for permutation entropy measurements. Int. J. Bifurcation Chaos 2007, 17, 3729-3733.

41. López-Ruiz, R.; Mancini, H.L.; Calbet, X. A statistical measure of complexity. Phys. Lett. A 1995, 209, 321-326.

42. The probability distribution $P$ is obtained once we fix the embedding dimension $D$ and the time delay $\tau$. The former parameter plays an important role for the evaluation of the appropriate probability distribution, since $D$ determines the number of accessible states, $D$ !, and tells us about the necessary length $N$ of the time series needed in order to work with a reliable statistics. In particular, Bandt and Pompe [38] suggest for practical purposes to work with $3 \leq D \leq 7$. Concerning this last point in all calculations reported here the condition $N \gg D$ ! is satisfied [40]. 
43. A flow of information takes place in both directions. However, the two flows do not have the same strength, i.e., more information is transferred from the classical to the quantal variables than vice versa. In this sense, we can assert that one variable is influencing the behavior of the other.

(c) 2010 by the authors; licensee Molecular Diversity Preservation International, Basel, Switzerland. This article is an open-access article distributed under the terms and conditions of the Creative Commons Attribution license http://creativecommons.org/licenses/by/3.0/. 